\title{
Influence of viscosity on the thermal energy produced in seismic fault: one-dimensional modeling
}

\author{
Guy Pascal Konga ${ }^{1}$, Fidèle Koumetio*,1, David Yemele ${ }^{1}$, Guy Bertrand Tanekou ${ }^{1}$ \\ (1) Unity of Research of Mechanics and Modelling of Physical Systems, Department of physics, Faculty of Science, \\ University of Dschang, PO Box 67Dschang, Cameroon
}

Article history: received December 12, 2018; accepted September 24, 2019

\begin{abstract}
This study aims to determine the thermal energy accumulation and the temperature distribution around a fault model when a slip weakening friction is imposed. We model the seismic fault sliding, also including viscosity, through a simplified one-body spring-slider model. We established the expression of thermal energy produced by slip-weakening friction in the case that we considered a simplified linearly slip-weakening friction law and a constant viscosity. Numerical simulation with Runge Kutta method is performed for determining the thermal energy in the case of linear slipweakening friction law, thermal-pressurization (TP) friction law, softening-hardening (SH) friction law and a simple slip-weakening (SW) friction law. The effect of the viscosity strongly modifies the results of the evolution of the temperature and the thermal energy. We obtained that the increase of temperature is due to the heat generated per unit volume and per unit time. We have showed that the temperature increases, attains a maximum value before decreasing and then, the system migrates toward a stationary state. In the fault, temperature distribution decrease when going far from the slip zone. This work establishes that the thermal energy is maximum at a moment when the amplitude of the seismic wave is large and that we describe as an instant of critical rupture of the seismic fault. Finally, we indicate that the effects of thermal energy on measurable physical quantities can be exploited to prevent the moment of critical rupture of the seismic fault.
\end{abstract}

Keywords: Earthquake dynamic, Slip-weakening friction, Viscosity, Heat generation, Temperature distribution.

\section{Introduction}

Earthquake is preceded by many natural phenomena, including the temperature anomaly. It is necessary to study the origin of the anomaly of temperature which is observed before a big earthquake. Many authors [Chester and Higgs, 1992; Fialko, 2004; Bizzarri and Cocco, 2006; Wang, 2006, 2007, 2009, 2011; Bizzarri, 2011a, 2011b; Bizzarri and Crupi, 2013] show that the thermal properties of the fault-zone rock are considered as the important code of thermal effect during earthquake rupture. Friction is one of most important factors in controlling the rupture processes of a seismic fault [Nur, 1978; Dieterich, 1979; Ruina, 1983; Cao and Aki, 1986; Knopoff et al., 


\section{Guy Pascal Konga et al.}

1992; Rice, 1993; Wang, 1996, 1997, 2007, 2012; Rubin and Ampuero, 2005; Ampuero and Rubin, 2008; Bizzarri, 2011c; Bhattachacharya and Rubin, 2014; Pelap et al., 2014]. Friction is also one of most important factors to evaluate the thermal energy produced in seismic fault before big earthquakes [Konga et al., 2017].

The calculation of the temperature distribution on the fault surface and its temporal evolution has been performed by many researches [Kato, 2001; Andrews, 2002; Fialko, 2004; Bizzarri and Cocco, 2006; Rice, 2006; Nielsen et al., 2008; Bizzarri, 2009a; Noda et al., 2009; Bizzarri 2010a, 2010b; Konga et al., 2017]. This temperature is due to frictional heat on a sliding interface. These works used the friction laws also known as constitutive laws, often motivated by laboratory experiments, determined the shear stress on the fault usually dependent on quantities such as the slip, velocity or other dynamic variables quantifying the internal state. The first works based on the fundamental rate- and- state dependent friction law which explained a large amount of laboratory data on rock friction were made by Dieterich [1979] and Ruina [1983]. The relations obtained by these authors constitute the basis of all the subsequent studies of the seismic cycle and have been further improved to incorporate the effect of temperature variations on friction coefficient [Chester and Higgs, 1992; Blanpied et al., 1998; Hirose and Shimamoto 2005; Di Toro et al., 2006; Beeler et al., 2007]. Kato [2001], Bizzarri and Cocco [2006] used the constitutive law to find the temperature distribution on the fault surface. Bizzarri and Cocco [2006] used both slip-weakening and rate- and state-dependent constitutive laws; in their work they employ the Linker and Dieterich evolution law for the state variable, and they couple the temporal variation of friction coefficient with those of effective normal stress. De Lorenzo and Loddo [2009] developed a numerical method to describe the thermo-mechanical evolution of the pre-seismic sliding phase which takes in account both the rate-, state- and temperature-dependent friction law.

Numerous studies used different constitutive relations to evaluate the temperature change in seismic fault. Recently many papers were published on the dynamic of earthquake [Wang, 2016; Wang, 2017a; Wang 2017b; Wang 2018] using the slip-weakening friction law. This slip-weakening friction law has not yet been used to evaluate the thermal energy and temperature distribution in seismic fault. Konga et al. [2017] established the first expression of thermal energy produced by friction forces in a seismic fault and explained the anomaly of temperature observed before a big earthquake without taking in account the effect of viscosity. They have considered friction forces only depending on velocity and frictional parameters which measures the homogeneousness of the surface between the two sides of the fault.

The viscosity plays an important role in the earthquake dynamics; it has been investigated theoretically and numerically by several authors. Jeffreys [1942] published the first paper which studies the effect of viscosity on the seismic fault. Schubert [1982] reported that the presence of water in fault, the variation of temperature and pressure change the viscosity. Rice et al. [2001] showed that the viscous effect is seen like an implicit factor on the friction law. Wang [2007] studied the viscous effect in the earthquake rupture. Numerous researchers [Knopoff et al. 1973, Xu and Knopoff 1994, Knopoff and Ni 2001, Dragoni and Santini, 2015] showed that the viscous effect is a factor in causing radiation to reduce energy during earthquake ruptures. Wang $[2016,2017 \mathrm{~b}]$ and Tanekou et al. [2018] studied the influence of viscosity on slip of sliders for one-degree-of-freedom spring-slider model. The influence of viscosity on the thermal energy produced in seismic fault has not yet been modeled. This statement brings us to the following question: what is the effect of this viscosity on temperature distribution?

In this work, we aim to study the role of viscosity on the thermal energy produced in the seismic fault by modelling this energy and temperature distribution in the framework of a slip-weakening friction law. This study is focused on slip of one-body dynamical spring-slider model in the presence of slip-weakening friction law initially proposed by Wang [2016]. We will start by the presentation of the slip-weakening friction law and displacement of slider according to the model that we are considering; thereafter we determine the thermal energy for a different form of slip-weakening friction law. Building on this, temperature distribution will be investigated.

\section{Description of physical model and force of friction}

We consider a model of earthquakes of figure 1a proposed by Wang [2016], where $m$ is the mass of the slider, $u$ is the displacement of slider, $K$ is the spring constant, $V_{p}$ is the speed of the driving force, $\eta$ is the coefficient of viscosity. 


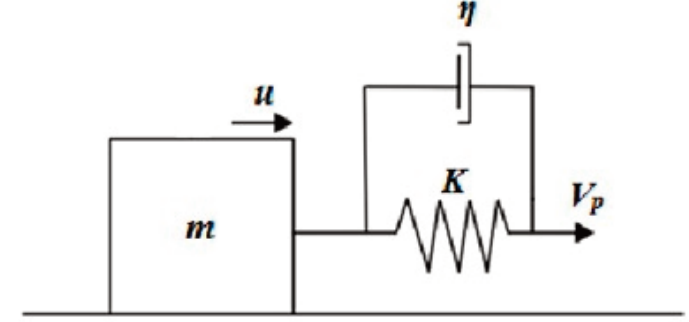

a)

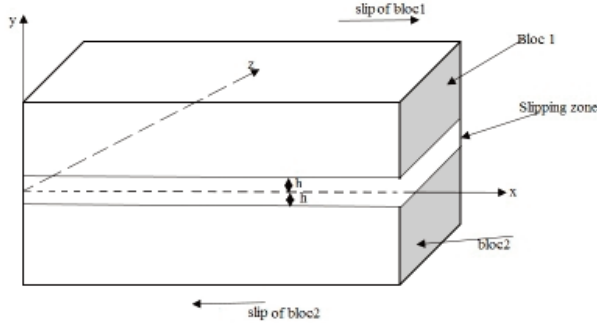

b)

Figure 1. a) One-body spring-slider model. b) Schematic of the model considered in the present study.

In the literature numerous researchers described several simple forms of friction laws. Burrigde and Knopoff [1967] introduced the first model of friction law used to study the earthquake dynamics. This model describes the seismic system as a collection of blocks resting on a surface with friction and which are connected to a set of springs. Byerlee [1978) shows that the friction depends on the time when both sides of the fault come into contact. Carlson and Langer [1989] present the dynamic of the fault like a chain of blocks connected by springs (the only non-linear element is the force of friction which exists between the blocks). That same year, they have proposed a purely non-linearity velocityweakening friction law which was also used by other authors [Carlson 1991; Carlson et al. 1991; Beeler et al. 2008]. To model the latter, they used the expression initially suggested by Burrigde and Knopoff [1967] while regarding the speed of the displacement of the blocks as variable. Pelap et al. [2014] added in the Burrigde and Knopoff model one coefficient which measures the homogeneousness of the surface between two sides of fault. Wang [1995, 1996, 2012] in this row considered a linearly velocity-dependent weakening-hardening friction, which is simplified from the friction law initially proposed by Burrigde and Knopoff [1967]. The weakening and hardening rate of dynamic friction with sliding velocity are two main parameters of this friction law. Cochard and Madariaga [1994] showed that, the velocity-dependent friction models drive at unphysical phenomena. This model of friction law is very unstable at low velocities both during the passage of the rupture front and during the possible slip arrest. Others friction laws are known to slip-weakening friction due to the thermal pressurization [Rice and Cocco, 2006]. More details of deepening description about thermal pressurization can be found in work of Sibson (1973], Rice [2006], Lachenbruch [1980], Raleigh and Everden [1981], Mase and Smith [1985, 1987], Lee and Delaney [1987], Andrews [2002].

In our study we use different slip-dependent friction laws described by Wang [2016] to model the thermal energy in the slipping zone and evaluate the temperature distribution, taking into account the viscosity. We consider the simplified linear slip-weakening friction law in the form of $F(u)=F_{0}-\gamma u$, where $\gamma$ is the weakening rate $F_{0}$, is the static force and $u$ the slip of slider in the analytical solution. For numerical simulations of the dynamic model, we used three slip-weakening friction laws: the thermal-pressurization (TP) friction law, the softening-hardening (SH) friction law and a simple slip-weakening (SW) friction law. A thermal pressurization friction law is defined by: $F(u)=F_{0} \exp (-u$ $/ u_{c}$ ), where $u_{c}$ is a characteristic displacement. In addition, the expression of the displacement softening-hardening friction law is: $F(u)=F_{0} \exp \left(-\left(u^{2}-u_{c}^{2}\right) / c^{2}\right)$, where $F_{0}, u_{c}$ and $c$ are the constant used by Cao and Aki $(1985,1986)$. $F_{0}, u_{c}$, and $c$ are respectively the static force, the characteristic displacement and a constant which has a dimensional of length. At the end, the simple slip-weakening friction law $\left[F(u)=F_{0} /\left(1+u / u_{c}\right)\right]$ is similar to the velocity-weakening friction law $\left[F(v)=F_{0} /\left(1+v / v_{c}\right)\right]$ (where $F_{0}$ is the statistic force and $v_{c}$ is the characteristic velocity and

$$
v=\frac{d u}{d t}
$$

is the velocity of the slider) proposed by Carlson and Langer [1989]. The characteristic displacement $u_{c}$ is controlled by some parameters like the fluid density, heat capacity, frictional strength, the thickness and the untrained pressurization factor of the fault zone [see Wang, 2018, and cited references therein]. The first three parameters are dependent of temperature. In this paper, we will consider that the characteristic displacement remains constant when the temperature varies because Wang [2018] gave the following indications:

- The observation data and theoretical analyses about the values of the fluid density during a seismic cycle are 


\section{Guy Pascal Konga et al.}

rare; its effect on characteristic displacement is neglected.

- Heat capacity is constant when the temperature is greater than $300^{\circ} \mathrm{K}\left(\approx 27^{\circ} \mathrm{C}\right)$ while the average ambient temperature of fault zone with depths ranging from 0 to $20 \mathrm{~km}$ is around $523^{\circ} \mathrm{K}\left(\approx 250^{\circ} \mathrm{C}\right)$; Hence, heat capacity cannot influence the characteristic displacement.

- A lack of long-term observations of frictional strength during a seismic cycle makes the study of its effect on earthquake recurrence impossible. For this reason, the effect of temperature on friction strength cannot be considered.

In this paper, we determine the slip and slip velocity from equation of motion. We used this slip and slip velocity to calculate the thermal energy (calculated as the integral of the product of slip velocity and slip-weakening friction law) and heat generated per unit volume and per unit time (calculated as the product of velocity and slip-weakening divided by thickness of slip zone). At the end we study the variation of temperature caused by the heat generated per unit volume and per unit time.

\section{Equation of motion}

In this paper we have used the equation of motion described by Wang [2016]. He considered the slip of a onebody dynamical spring-slider model. This equation of motion of slider is given by:

$$
m \frac{d^{2} u}{d t^{2}}=-K\left(u-v_{p} t\right)-F(u, v)-\Phi(v)
$$

Where $F$ is a frictional force between the slider and the background ( $F$ could be a function of $u$ and $v$ ). $F$ is controlled by several factors like displacement of slider, the characteristics displacements and other parameters which can be seen in the paper of Wang [2016], $\Phi=\eta v$ is the viscous force between the slider and the background, $\eta$ is the coefficient of viscosity. More details deepening description about viscosity and viscous force can be found in Wang [2016]. The viscosity coefficient of rocks is controlled by the temperature. In this work, we considered the simplified expression

$$
\eta=\eta_{0} \exp \left(\beta\left(1-\frac{T-T_{C}}{T_{H}-T_{C}}\right)\right)
$$

where $\eta_{0}$ is a reference value, $\beta$ is a constant, $T_{C}$ is the steady coldest temperature of the side walls and $T_{H}$ is the hottest temperature. Diniega et al. [2013] assume that $T_{C}=1050^{\circ} \mathrm{C}$ and $T_{H}-T_{C}=100^{\circ} \mathrm{C}$ [more details are found in Diniega et al., 2013 and Wang, 2017a]. The slider is pulled by a leaf spring of strength, $K$, with a constant velocity, $V_{p}$, which represents the speed of a moving plate. When the driving force, $K V_{p} t$, is slightly larger than the static frictional force $F_{0}$, friction changes from static frictional strength to dynamic one.

\section{Work of friction force and thermal energy}

In this part, we shall calculate the work of friction force for several cases. In the first time we use the simplified linearly slip-weakening friction law (considering on the one hand that the viscosity remains constant, which makes it possible to have an analytical solution and on the other hand that this viscosity varies with the temperature), secondly we use TP, SH, and SW friction law (considering that the viscosity varies with the temperature). The thermal energy is generated within the slipping zone (Figure 1b). The work done due to frictional forces is defined by the scalar product of this force and displacement of slider. The general expression is given by:

$$
W_{f}=\int F(u) d u=\int_{0}^{t} F(u) \frac{d u}{d \tau} d \tau=\int_{0}^{t} F(u) v d \tau
$$

We integrated equation (2) by taking $\mathrm{t}=0$ as the period where the accumulation of energy starts. 


\subsection{Constant viscosity}

In this case, we considered only the simplified linear slip-weakening friction law to determine the analytical expression of thermal energy.

From the analytical study, the general expression of the displacement of slider, $u(t)$, is given by Wang [2016]

$$
u(t)=\left(v_{0} / \omega_{n}\right) \exp \left(-\eta_{0} t / 2 m\right) \sin \left(\omega_{n} t\right)
$$

where $\omega_{n}=\left[\omega_{0}^{2}-\left(\eta_{0}^{2}-4 m \gamma\right) / 4 m^{2}\right]^{1 / 2}, \omega_{0}=(K / m)^{1 / 2}, \omega_{n}$ is composed by a part $\omega_{0}$ only depending on the spring-slider system features and the other part depending on friction and viscosity of the interface, the ration $\left(v_{0} / \omega_{n}\right)$ denoted the amplitude of slip function and varies with four model parameters, $\eta_{0}$ is the coefficient of viscosity, $v_{0}$ is the initial velocity and $\gamma$ is the weakening rate.

From equation (3) the velocity of slider $v$ is given as:

$$
v=v_{0}\left(-\frac{\eta_{0} t}{2 m \omega_{n}} \sin \left(\omega_{n} t\right)+v_{0} \cos \left(\omega_{n} t\right)\right) \exp \left(-\frac{\eta_{0} t}{2 m}\right)
$$

The simplified linear slip-weakening friction law defined as:

$$
F(u)=F_{0}-\gamma u
$$

The work done by this friction is obtained as:

$$
W_{f}=A_{1}\left[\left(A_{2} \sin \left(\omega_{n} t\right)-A_{3} \cos \left(\omega_{n} t\right)\right) \exp (-\alpha t)+A_{5}\right]-S_{1}\left[\left(S_{2} \sin \left(2 \omega_{n} t\right)-S_{3} \cos \left(2 \omega_{n} t\right)\right) \exp (-2 \alpha t)+S_{4}\right]+\frac{A_{4}}{2 \alpha}(1-\exp (-2 \alpha t))
$$

where $\alpha, \mathrm{S}_{1}, \mathrm{~S}_{2}, \mathrm{~S}_{3}, \mathrm{~S}_{4}, \mathrm{~A}_{1}, \mathrm{~A}_{2}, \mathrm{~A}_{3}, \mathrm{~A}_{4}$ and $\mathrm{A}_{5}$ are constants defined by the following expression:

$$
\begin{gathered}
\alpha=\frac{\eta_{0}}{2 m}, \quad S_{1}=\frac{\alpha}{2\left(\alpha^{2}+\omega_{n}^{2}\right)}, \quad S_{2}=\frac{\gamma \omega_{n} A^{2}}{2}(\alpha+1), \quad A=\frac{v_{0}}{\omega_{n}}, \quad S_{3}=\frac{\gamma A^{2}}{2}\left(\alpha+\omega_{n}^{2}\right), \quad S_{4}=\frac{\gamma A^{2}}{2}\left(\omega_{n}^{2}-\alpha\right), \\
A_{1}=\frac{F_{0}}{\alpha^{2}+\omega_{n}^{2}}, A_{2} S_{1} \omega_{n}-S_{2} \alpha, \quad A_{3}=S_{2} \omega_{n}+S_{1} \alpha, \quad A_{4}=\frac{\gamma \alpha A^{2}}{2}, \quad A_{5}=S_{2} \omega_{n}+S_{1} \alpha
\end{gathered}
$$

Thermal energy due to the friction is defined as the absolute value of the work done by frictional forces and is defined by:

$$
Q(t)=\left|W_{f}\right|
$$

We have performed a MATLAB simulation to obtain Figure 2a which shows the distribution of thermal energy accumulated by friction at the level of the fault in presence of viscosity as well as Figure $2 b$ which shows the evolution of slip according to time for simplified linear slip-weakening friction law. 


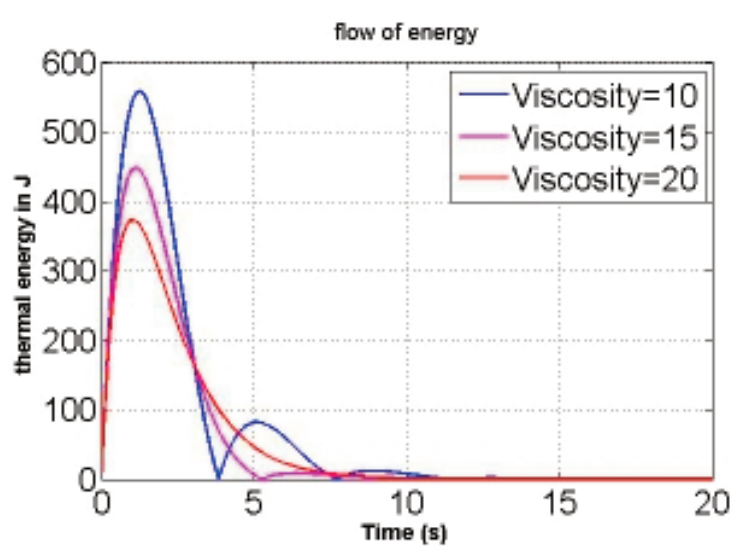

a)

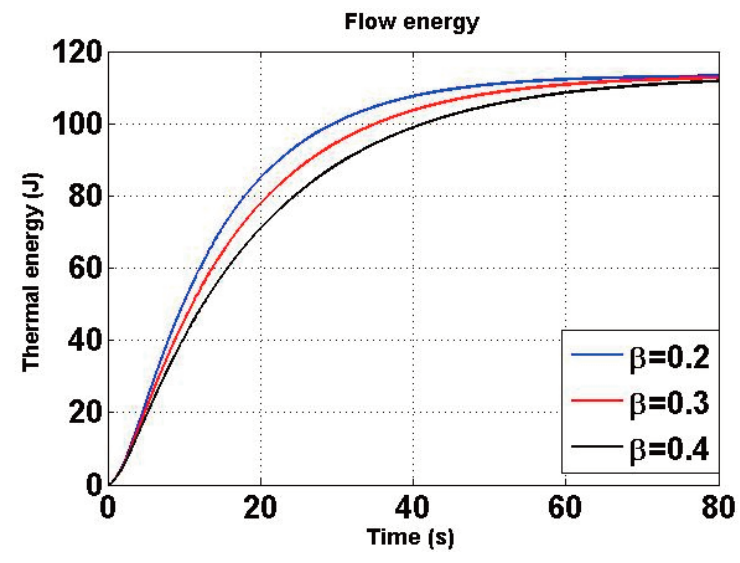

c)

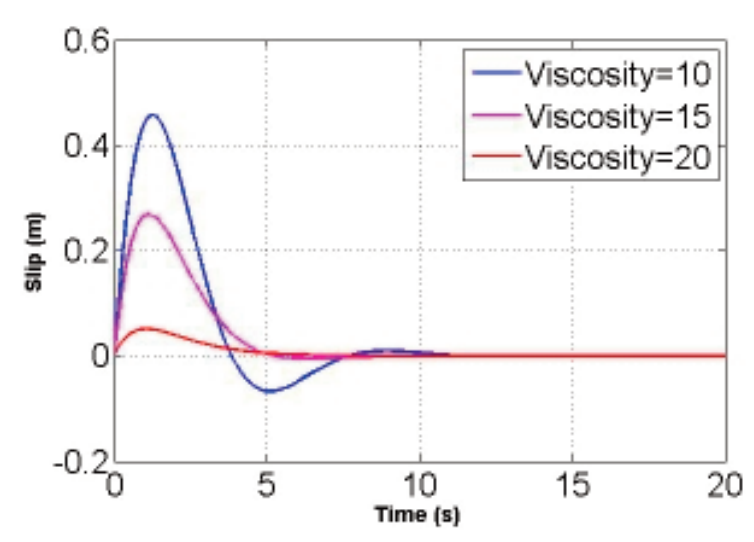

b)

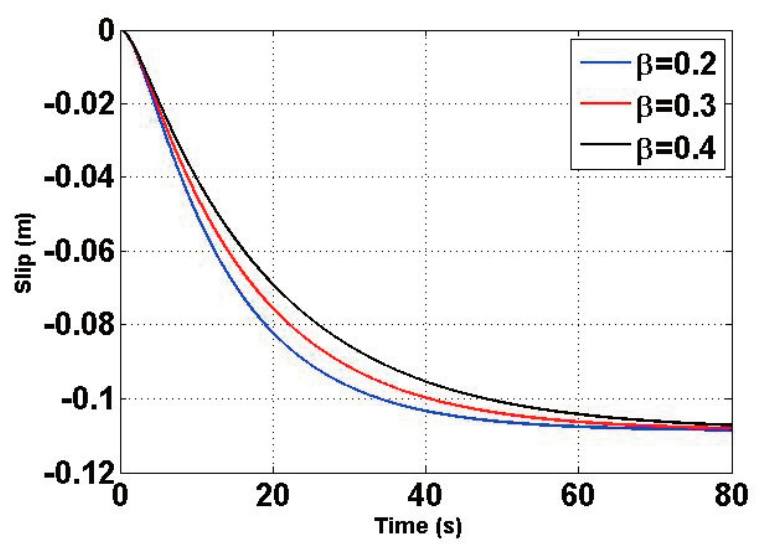

d)

Figure 2. a) Evolution of thermal energy according to time due to the simplified linearly slip-weakening friction law in the fault when the viscosity is constant. b) Evolution of slip according to time due to the simplified linearly slipweakening friction law in the fault when the viscosity is constant. c) Evolution of thermal energy according to time due to the simplified linearly slip-weakening friction law in the fault when the viscosity varies with temperature. d) Evolution of slip according to time due to the simplified linearly slip-weakening friction law in the fault when the viscosity varies with temperature. Values used: $\eta_{0}=10 \mathrm{~N} /\left(\mathrm{m}^{2} / \mathrm{s}\right), \mathrm{V}_{\mathrm{p}}=10^{-11} \mathrm{~m} / \mathrm{s}, \gamma=0.8 \mathrm{~N} / \mathrm{m}$, $\mathrm{K}=10 \mathrm{~N} / \mathrm{m}, \mathrm{m}=10 \mathrm{~kg}$ and $\mathrm{F}_{0}=1$.

\subsection{Temperature dependent viscosity}

Knowing that

$$
\eta=\eta_{0} \exp \left(\beta\left(1-\frac{T-T_{C}}{T_{H}-T_{C}}\right)\right)
$$

the viscosity varies with the temperature when $\beta \neq 0$. We considered in this case all the slip-weakening friction law listed above.

For the simplified linear slip-weakening friction law, equation (1) can be rewritten as two first-order differential equations and we added in this system the third equation which gives the thermal energy generated by simplified linear slip-weakening friction law and the system is given as: 


$$
\left\{\begin{array}{l}
\frac{d u}{d t}=v \\
\frac{d u}{d t}=-\frac{1}{m}\left((K-\gamma) u+\eta_{0} \exp \left(\beta\left(1-\frac{T-T_{C}}{T_{H}-T_{C}}\right)\right) v+F_{0}-K V_{p} t\right) \\
\frac{d W_{L}}{d t}=v\left(F_{0}-\gamma u\right) \\
\text { at } t=0, \text { we have } W_{L}=0
\end{array}\right.
$$

Since the viscosity is temperature dependent, the heat conduction equation (9) is used to derive it distribution. The heat conduction equation is given by:

$$
\frac{\partial T}{\partial t}=\frac{k}{\rho c_{s}} \frac{\partial^{2} T}{\partial y^{2}}+\frac{q(t)}{\rho c_{s}}
$$

where $k$ is the thermal conductivity, $c_{s}$ the specific heat of rock at the constant pressure, $\rho$ is the density of rock (Fialko, 2004) and $\gamma$ represents the spatial coordinate normal to the fault. This system is semi-infinity medium at the initial temperature distribution on the fault plane $T_{0}$. In equation (9) $q$ is the heat generated per unit volume and per unit time $\left([q]=\mathrm{W} / \mathrm{m}^{3}\right)$ i.e. the power developed by friction. The volume is defined by $V=S(2 h)$, we have taken the area $S$ equal at an unit in this work and $2 h$ is the thickness of the slip zone (see Figure $1 \mathrm{~b}$ ). Bizzarri and Cocco (2006), Rice (2006) show that the heat source is uniformly generated in slip zone layer:

$$
q(t)= \begin{cases}\frac{F(u) v}{2 h}, & t \geq 0,|y| \leq h \\ 0, & |y| \geq h\end{cases}
$$

The slip velocity $v$ and slip $u$ in equation (10) are obtained by resolving the equation (1) for each friction law.

We used the Runge Kutta method to solve this system and we have performed a MATLAB simulation to obtain the curves of Figure 2c which shows the distribution of thermal energy as well as Figure 2d which shows the evolution of slip according to time considering the simplified linear slip-weakening friction law when the viscosity varies with the temperature.

For TP friction law, we have a system (11):

$$
\left\{\begin{array}{l}
\frac{d u}{d t}=v \\
\frac{d u}{d t}=-\frac{1}{m}\left(K u+\eta_{0} \exp \left(\beta\left(1-\frac{T-T_{C}}{T_{H}-T_{C}}\right)\right) v+F_{0} \exp \left(-u / u_{c}\right)-K V_{p} t\right) \\
\frac{d W_{T P}}{d t}=v F_{0} \exp \left(-u / u_{c}\right) \\
\text { at } t=0, \text { we have } W_{T P}=0
\end{array}\right.
$$

We obtained the curves of Figure 3a which gives the distribution of thermal energy as well as Figure $3 \mathrm{~b}$ which shows the evolution of slip according to time considering the TP friction law.

For the SH friction law, we have a system (12): 


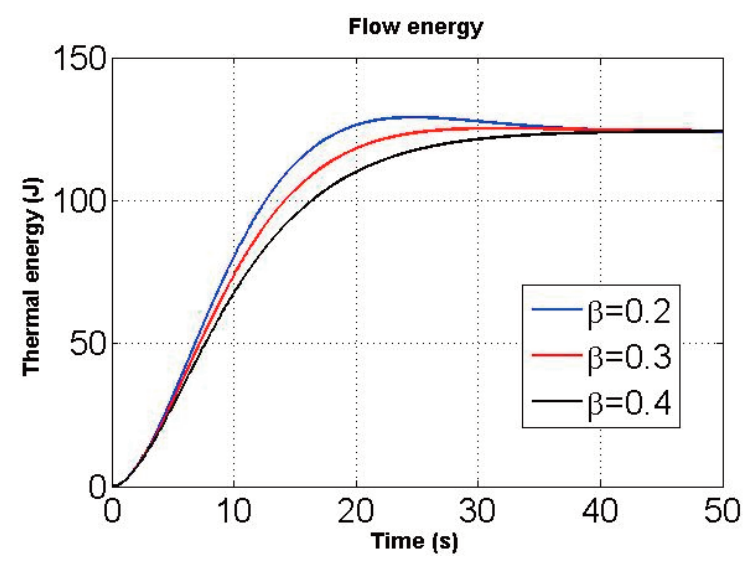

a)

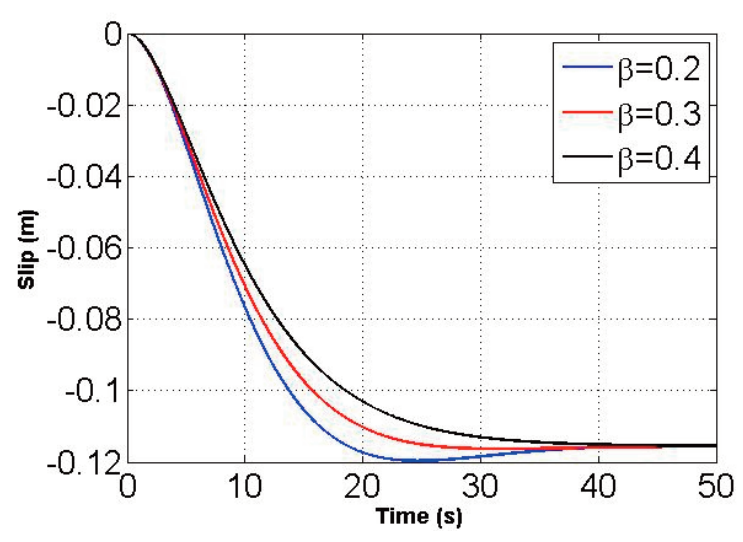

b)

Figure 3. a) Evolution of thermal energy according to time due to the TP friction law in the fault. b) Evolution of slip according to time due to the TP friction law in the fault. Values used: $\mathrm{K}=10 \mathrm{~N} / \mathrm{m}, \mathrm{m}=10 \mathrm{~kg}, \mathrm{~V}_{\mathrm{p}}=10^{-11} \mathrm{~m} / \mathrm{s}, \eta_{0}=$ $10 \mathrm{~N} /\left(\mathrm{m}^{2} / \mathrm{s}\right), \mathrm{u}_{\mathrm{c}}=0.5, \mathrm{~F}_{0}=1$.

$$
\left\{\begin{array}{l}
\frac{d u}{d t}=v \\
\frac{d u}{d t}=-\frac{1}{m}\left(K u+\eta_{0} \exp \left(\beta\left(1-\frac{T-T_{C}}{T_{H}-T_{C}}\right)\right) v+F_{0} \exp \left(-\frac{\left(u^{2}-u_{c}^{2}\right)}{c^{2}}\right)-K V_{p} t\right) \\
\frac{d W_{S H}}{d t}=v F_{0} \exp \left(-\frac{\left(u^{2}-u_{c}^{2}\right)}{c^{2}}\right) \\
\text { at } t=0, \text { we have } W_{S H}=0
\end{array}\right.
$$

We obtained the curves of Figure 4a which gives the distribution of thermal energy as well as Figure 4b which shows the evolution of slip according to time considering the SH friction law.

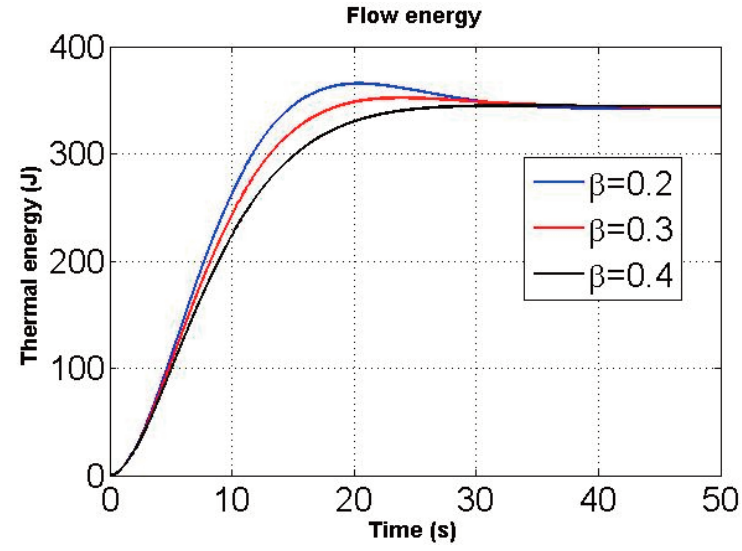

a)

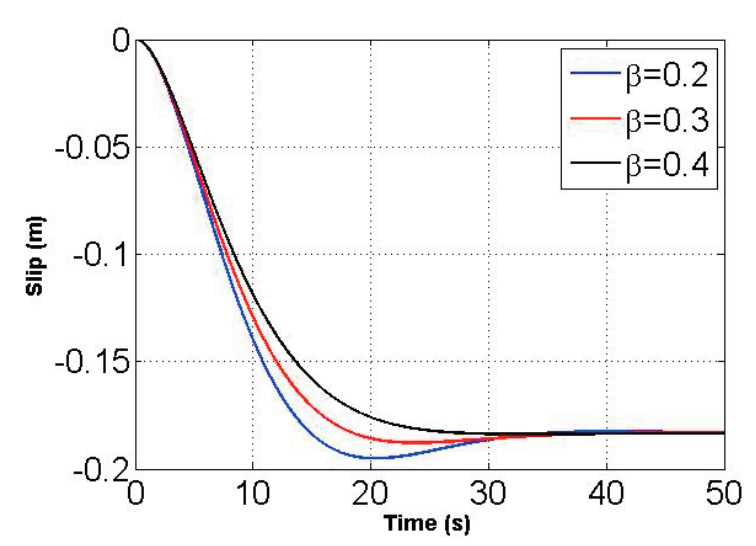

b)

Figure 4. a) Evolution of thermal energy according to time due to the SH friction law in the fault. b) Evolution of slip according to time due to the SH friction law in the fault. Values used: $\mathrm{K}=10 \mathrm{~N} / \mathrm{m}, \mathrm{m}=10 \mathrm{~kg}, \mathrm{~V}_{\mathrm{p}}=10^{-11} \mathrm{~m} / \mathrm{s}, \eta_{0}=$ $10 \mathrm{~N} /\left(\mathrm{m}^{2} / \mathrm{s}\right), \mathrm{u}_{\mathrm{c}}=0.5, \mathrm{~F}_{0}=1$. 
For the SW friction law, we have a system (13)

$$
\left\{\begin{array}{l}
\frac{d u}{d t}=v \\
\frac{d u}{d t}=-\frac{1}{m}\left(K u+\eta_{0} \exp \left(\beta\left(1-\frac{T-T_{C}}{T_{H}-T_{C}}\right)\right) v+F_{0}\left(\frac{1}{1+u / u_{c}}\right)-K V_{p} t\right) \\
\frac{d W_{S W}}{d t}=v F_{0} \frac{1}{1+u / u_{c}} \\
\text { at } t=0, \text { we have } W_{S W}=0
\end{array}\right.
$$

We obtained the curves of Figure 5a which gives the distribution of thermal energy as well as Figure 5b which shows the evolution of slip according to time considering the SW friction law.

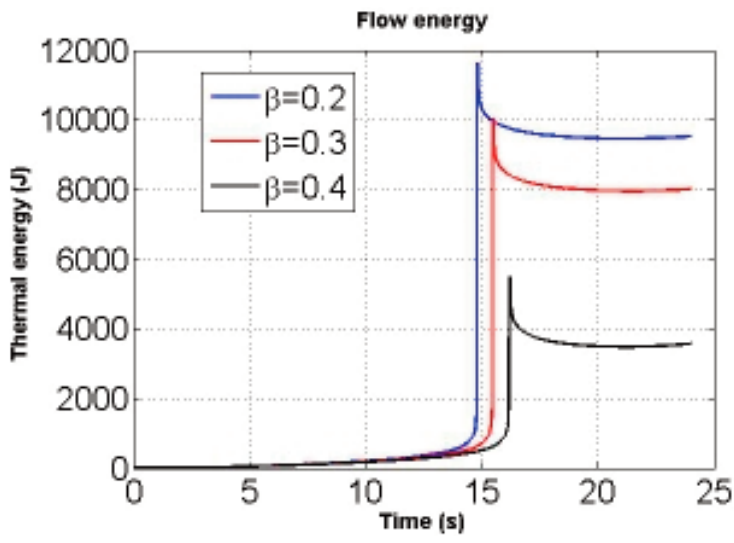

a)

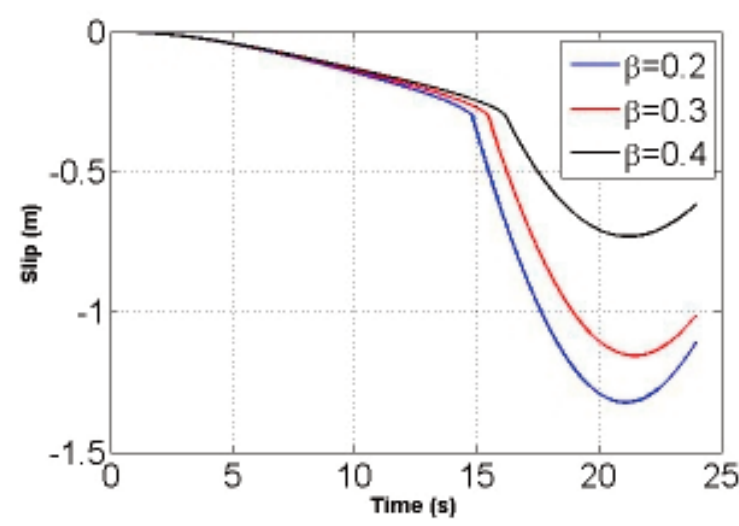

b)

Figure 5. a) Evolution of thermal energy according to time due to the SW friction law in the fault. b) Evolution of slip according to time due to the SW friction law in the fault. Values used: $\mathrm{K}=10 \mathrm{~N} / \mathrm{m}, \mathrm{m}=10 \mathrm{~kg}, \mathrm{~V}_{\mathrm{p}}=10^{-11} \mathrm{~m} / \mathrm{s}, \mathrm{\eta}_{0}=$ $10 \mathrm{~N} /\left(\mathrm{m}^{2} / \mathrm{s}\right), \mathrm{u}_{\mathrm{c}}=0.5, \mathrm{~F}_{0}=1$.

The observation of Figure 2a reveals that for the simplified linear slip-weakening friction law and for a constant viscosity, the thermal energy abruptly increases, reaches a maximum value and then abruptly decreases in this case of an analytical solution. It can also be seen that the maximum value of the thermal energy decreases as the viscosity increases, this maximum value is reached almost at the same moment whatever the value of the viscosity. Figure $2 \mathrm{~b}$ shows that for the low value of the viscosity $(\eta=10)$, the direction of movement of the block changes twice (approximately at times 2.9s and 7.5s). This curve shows that the amplitude of the movement decreases with time, from one phase of the movement to another until a zero amplitude. We finally observe that the absolute value of the slip varies exactly in the same way as the thermal energy produced in the seismic fault whatever the value of the viscosity.

The observation of Figure $2 \mathrm{c}$ reveals that for the simplified linear slip-weakening friction law and for a viscosity that varies with the temperature $(\beta \neq 0)$, the thermal energy regularly increases until it reaches an asymptotic value in this case of a purely numerical solution. Figure $2 \mathrm{c}$ also shows that the slope of the curve decreases when the viscosity values are larger, and the asymptotic value is nearly the same for all curves. Figure $2 \mathrm{~d}$ shows that the absolute value of the slip varies exactly in the same way as the thermal energy produced in the seismic fault.

Figures 3a, 3b, 4a and 4b show that for TP and SH friction law, the evolutions of the curves are exactly the same as for the simplified linear slip-weakening friction law in the case of a purely numerical solution for thermal energy as well as for slipping according to time. 


\section{Guy Pascal Konga et al.}

Figure 5a shows that for SW friction law, the thermal energy increases along a practically vertical slope after a certain amount of time, reaching a maximum value, then slightly decreases along the same slope before keeping a constant value. In this case, the curve of variation of the absolute value of the slip as a function of time (Figure 5b) regularly increases until reaching a point of inflection at the moment when the thermal energy is maximum, then it continues to increase along a steeper slope, reaches the maximum value and starts decay. We notice that the variation of the viscosity influences the slip and the thermal energy produced in the seismic fault for the simplified linear slip-weakening, TP, SH and SW friction law.

\section{Temperature distribution}

We compute the temperature changes caused by heat generated per unit volume and per unit time due to slipweakening friction law in seismic fault. We considered the heat generated per unit volume and per unit time as a heat-flux in the 1-D heat conduction equation given by (9). This heat-flux takes in to account the effect of viscosity. For each friction law we have evaluated the heat generated per unit volume and per unit time by the following equations:

For the linearly slip-weakening friction law

$$
q(t)= \begin{cases}\frac{\left(F_{0}-\gamma u\right) v}{2 h}, & t \geq 0,|y| \leq h \\ 0, & |y| \geq h\end{cases}
$$

For the TP friction law, we have the following equation

$$
q_{T P}(t)= \begin{cases}\frac{F_{0} \exp \left(-u / u_{c}\right) v}{2 h}, & t \geq 0,|y| \leq h \\ 0, & |y| \geq h\end{cases}
$$

For the SH and SW friction law, we have respectively the equations (16) and (17)

$$
\begin{aligned}
& q_{S T}(t)= \begin{cases}\frac{F_{0} \exp \left(-\frac{\left(u^{2}-u_{c}^{2}\right)}{c^{2}}\right) v}{2 h}, & t \geq 0,|y| \leq h \\
0, & |y| \geq h\end{cases} \\
& q_{S W}(t)= \begin{cases}\frac{F_{0} \exp \left(\frac{1}{1+u / u_{c}}\right) v}{2 h}, & t \geq 0,|y| \leq h \\
0, & |y| \geq h\end{cases}
\end{aligned}
$$

Figures 6a-d illustrate the time variation of heat generated per unit volume and by unit time for the simplified linear slip-weakening, TP, SH and SW friction law. Using the analytical expression (equation 10) and numerical results of the heat generated per unit volume and per unit time (equations 14, 15, 16 and 17), equation 9 is solved numerically with the help of the finite difference method.

We have numerically simulated the solution of 1-D heat conduction equation. Results of these calculation are illustrated in Figures 7a-f. In Figures $7 \mathrm{a}$ and $7 \mathrm{~b}$, we used only the linearly slip-weakening friction law in the case of constant viscosity; temperature distribution is monitored simultaneously at several spaces and times. In these tests, we vary distance when the time is fixed and then the time when the distance is fixed. Figure 7a illustrates a spatiotemporal evolution of temperature distribution (or temperature distribution versus distance $\mathrm{y} / \mathrm{h}$ for a series of constant time) on the slipping fault surface. It shows that the temperature variation is maximum at the slipping 


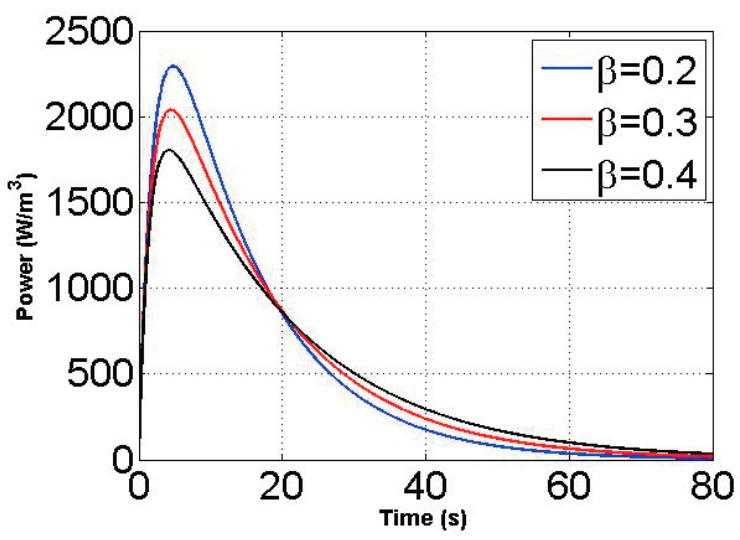

a)

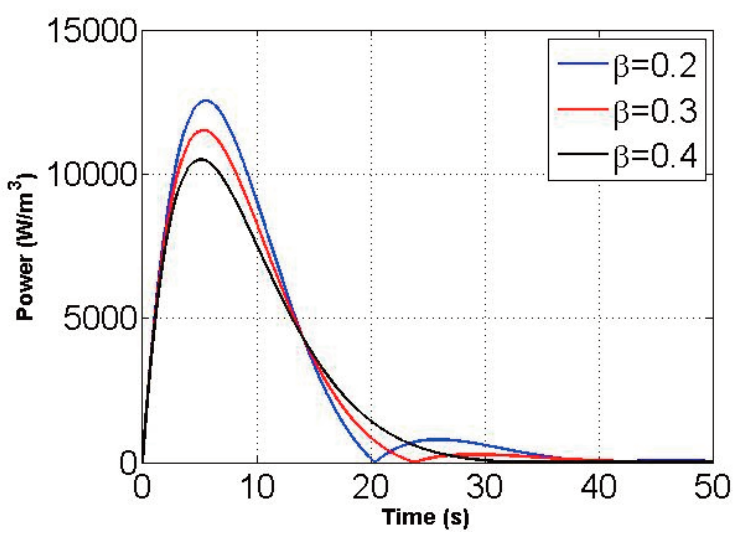

c)

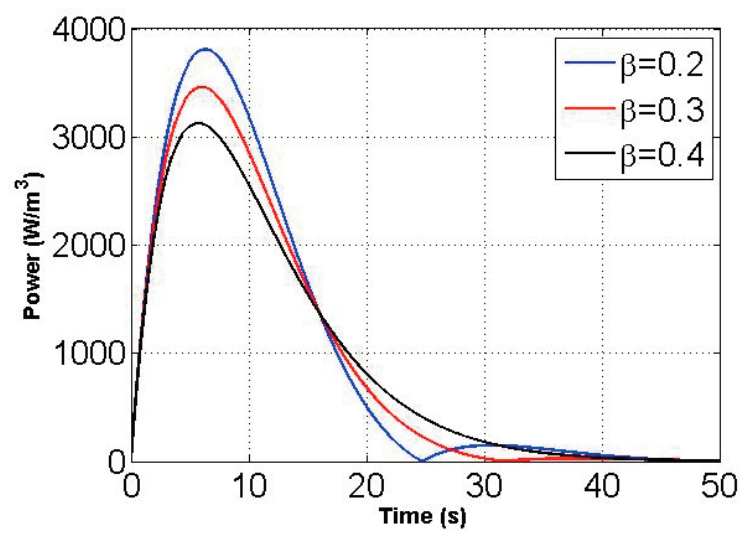

b)

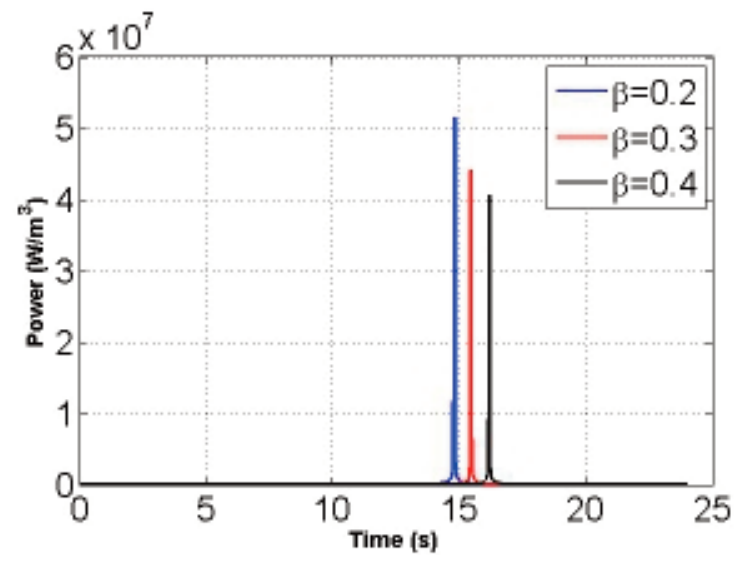

d)

Figure 6. a) Evolution of heat generated per unit volume and per unit time due to the linearly slip-weakening friction law in the fault when the viscosity varies with temperature. b) Evolution of heat generated per unit volume and per unit time due to the TP friction law in the fault. c) Evolution of heat generated per unit volume and per unit time due to the SH friction law in the fault. d) Evolution of heat generated per unit volume and per unit time due to the SW friction law in the fault. Values used: $\mathrm{K}=10 \mathrm{~N} / \mathrm{m}, \mathrm{m}=10 \mathrm{~kg}, \mathrm{~V}_{\mathrm{p}}=10^{-11} \mathrm{~m} / \mathrm{s}, \eta_{0}=10 \mathrm{~N} /\left(\mathrm{m}^{2} / \mathrm{s}\right), \mathrm{u}_{\mathrm{c}}=0.5, \mathrm{~F}_{0}=1$.

zone and decreases monotonically when moving away from this zone. The results presented in Figure $7 \mathrm{~b}$ confirm that the maximum value of the temperature variation decreases with the distance from the fault zone (we can see from this figure that by increasing the distance from the slipping zone, the temperature variation progressively decreases). Figure $7 \mathrm{~b}$ also shows that the time required to reach the maximum value of the temperature variation remains the same for all distances. Figures $7 \mathrm{c}$-e, illustrate the temperature distribution in semi-infinity medium obtained from the heat generated per unit volume and per unit time produced by the different slip-weakening friction law (linearly slip-weakening, TP, SH and SW friction law) when the viscosity varies with the temperature. Figures 7c-e (for linearly slip-weakening, TP and SH friction law respectively) show that the temperature variation increases abruptly to a maximum value then decreases with a slope that gradually decreases until reaching zero; this maximum value decreases with the increasing viscosity. In Figure $7 \mathrm{f}$ (for SW friction law), the temperature increases after a certain amount of time, reaching a maximum value and then decreases; the time at which this maximum value is attained increases with the increasing viscosity. 


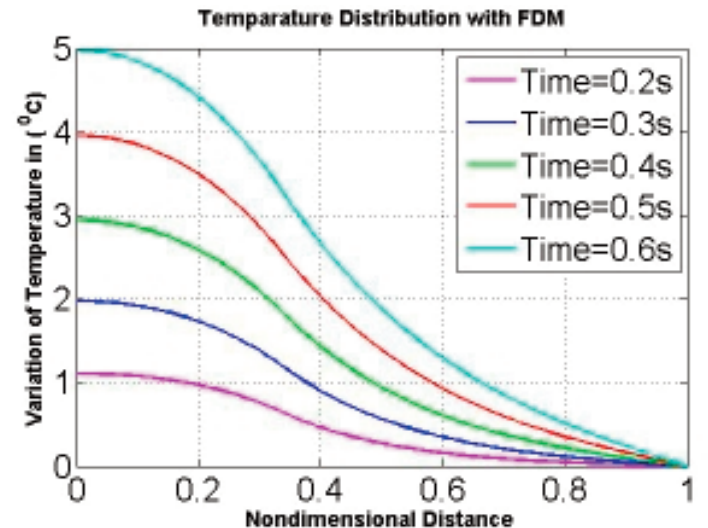

a)

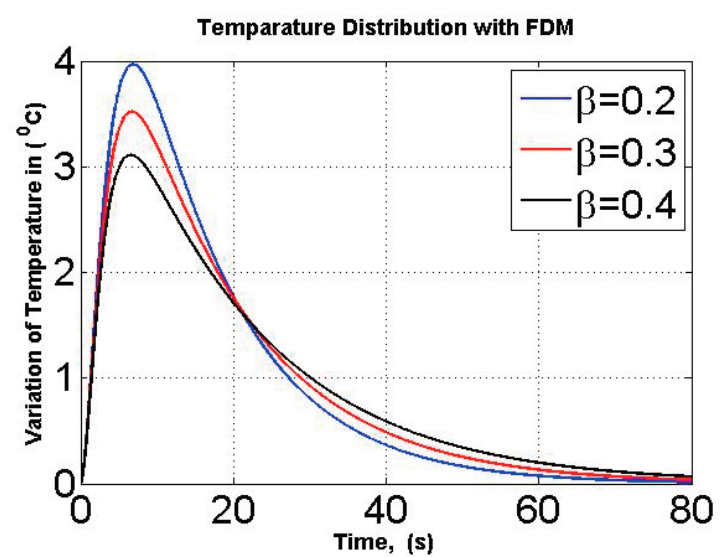

c)

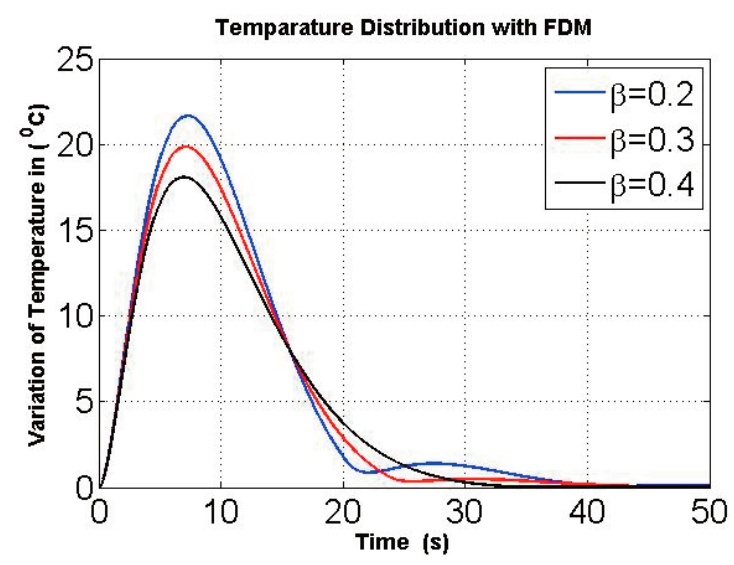

e)

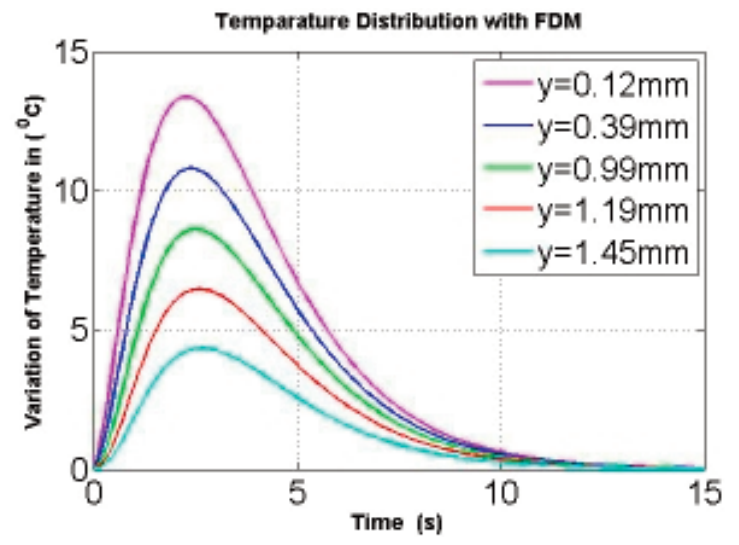

b)

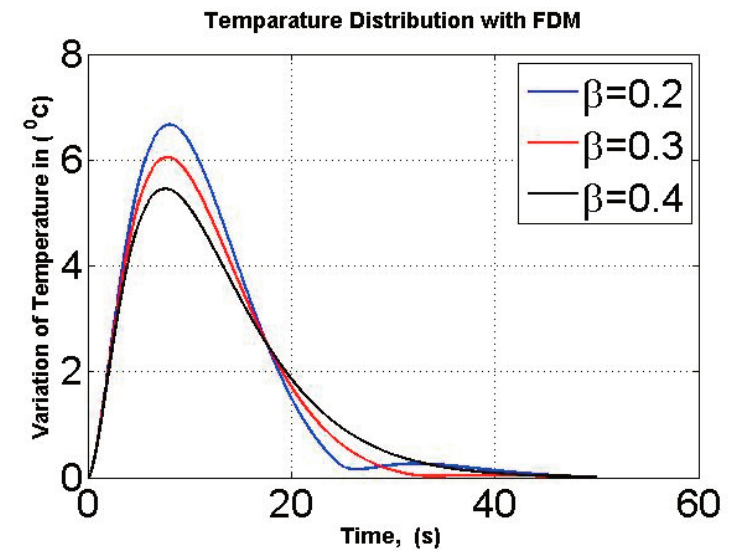

d)

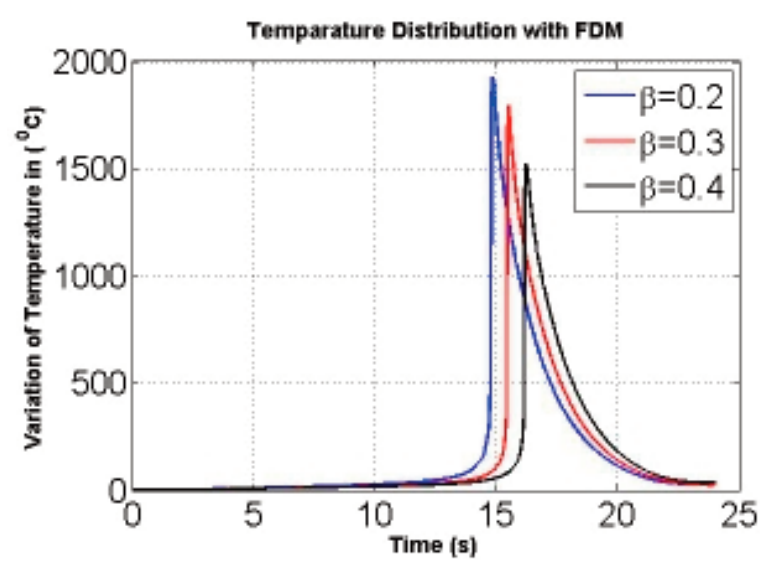

f)

Figure 7. a) Variation of temperature according to nondimentional distance due to the simplified linearly slip-weakening friction law in the fault when the viscosity is constant. b) Times series of temperature distribution in semiinfinity medium for simplified linearly slip-weakening friction law in the fault when the viscosity is constant. c) Times series of temperature distribution in semi-infinity medium for simplified linearly slip-weakening friction law in the fault when the viscosity varies with temperature. d) Times series of temperature distribution in semiinfinity medium for TP friction law in the fault. e) Times series of temperature distribution in semi-infinity medium for SH friction law in the fault. f) Times series of temperature distribution in semi-infinity medium for SW friction law in the fault. Values used: $\mathrm{K}=10 \mathrm{~N} / \mathrm{m}, \mathrm{m}=10 \mathrm{~kg}, \mathrm{~V}_{\mathrm{p}}=10^{-11} \mathrm{~m} / \mathrm{s}, \eta_{0}=10 \mathrm{~N} /\left(\mathrm{m}^{2} / \mathrm{s}\right), \mathrm{u}_{\mathrm{c}}=0.5$, density: $\rho=2700 \mathrm{~kg} / \mathrm{m}^{3}$, heat capacity: $\mathrm{c}_{\mathrm{s}}=1000 \mathrm{~J} / \mathrm{kg}$, thermal conductivity: $\mathrm{k}=2.7 \mathrm{~W} / \mathrm{m}^{\circ} \mathrm{c}, \mathrm{F}_{0}=1$. 


\section{Discussion}

In this paper we have analytically (when the viscosity is constant) and numerically (when the viscosity varies with the temperature) determined the thermal energy due to slip-weakening friction in the presence of viscosity and we have numerically solved the 1-D thermal conduction equation. We indicate that this was done so because of the difficulty of finding an analytical solution to the differential equations obtained when the viscosity varies with the temperature. In the case where the viscosity does not vary with the temperature, the maximum of the absolute value of the slip decreases when the viscosity increases (Figure 2b), which corroborates the result obtained by Wang [2016]. In the case where the viscosity varies with the temperature, for linearly slipweakening, TP and SH friction law, the curves of variation of the slip with time (Figures 2d, 3b, $4 b$ and $5 b$ ) have almost the same appearance as those obtained by Wang [2017] who studied the slip of a two-degree-of-freedom spring-slider model, consisting of two sliders, in the presence of slip-dependent friction due to thermal pressurization and viscosity. Since (1) slip also depends on the frictional force, (2) the heat energy is a function of both the friction force and the slip, it is normal that the curves of variation of thermal energy and the absolute value of the slip according to time have the same appearance.

In all cases (Figures $7 \mathrm{~b}-\mathrm{f}$ ), the temperature variation abruptly increases to a maximum value then decreases with a slope that gradually decreases until reaching zero, which means that the system converges to a stationary state. These results are comparable to those ones obtained by Kato [2001], Fialko [2004], Bizzarri [2010c] and Bizzarri [2014] with regard to the variation of temperature as a function of time. We find that in the context of our study, as the system converges to a stationary state, the thermal energy and the slip remain constants. We think that the stationary state describes the uniform motion of the blocks corresponding to an aseismic creep along the fault.

Fialko [2004] shows that the effective thickness of the earthquake slip zone controls not only the magnitude of temperature increase, but also maximum temperature in the fault. Our model (Figures 7a-b) gives the same observation, but the attenuation of the temperature distribution is more pronounced when one moves away from slip zone. Bizzarri [2010] added that the more general solution found by Bizzarri and Cocco [2006] by using both slip weakening and rate- and state-dependent constitutive relations can be reconciled with solution of Kato [2001] found by using rate-, state- and temperature- dependent friction law when the slipping zone halfthickness (w) is smaller than $10 \mu \mathrm{m}$. It appears that in our model where we use the slip-weakening friction law, the magnitude of temperature increase and the maximum temperature are not only controlled by a thickness of slip zone (Figures 7a-b), but also by the viscosity (Figures 7c-f).

Bizzarri and Cocco [2006] report that, before the rupture starts the temperature is equal to its initial value and after crack start the temperature increases, depending on the value of the slipping zone thickness. In this case, we can understand that they suppose that there is not slip or displacement before the earthquake. If the temperature remained equal to its initial value, one cannot observe the temperature anomaly before an earthquake, while our study as well as those of the other authors [Saraf and Choudhury, 2004; Tramutoli et al., 2005; Rezapour et al, 2008; Qin et al., 2012] establishes well this temperature anomaly.

Figures $2 \mathrm{a}$ to $4 \mathrm{~b}$ show that the thermal energy produced in the seismic fault is maximum at the same time as the absolute value of the slip while Figures $5 \mathrm{a}$ and $5 \mathrm{~b}$ show that the thermal energy is maximum at a time $\mathrm{t}_{\mathrm{c}}$ corresponding to the point of inflection on the slip curve. In this last case, we can think that there are several phases of the movement since the slip continues to increase after the instant $t_{c}$ (Figure $5 b$ ), which can be understood with the reality of the replicas during the earthquakes. However, the thermal energy remains practically constant after the instant $t_{c}$ (Figure $5 a$ ), a phenomenon that remains to be explained in the case of SW friction law. Focusing on the first phase of the movement, it appears that at the end of this phase, thermal energy as well as the absolute value of the slip has its greatest value for linearly slip-weakening, TP, SH and SW friction law. When the absolute value of the slip is great, the amplitude of the seismic wave is large, that implies a higher risk of enormous damage. We can therefore say that the thermal energy is maximal at a critical moment that we describe as a time of critical rupture of the seismic fault. Knowing that thermal energy influences measurable physical quantities such as geothermal flow, electrical resistivity of rocks, the effects of this thermal energy can be exploited to prevent the moment of critical rupture of the seismic fault. 


\section{Guy Pascal Konga et al.}

\section{Conclusions}

This paper investigated the effect of viscosity parameter on the thermal energy produced in the seismic fault via a one-body dynamical spring-slider model with slip-weakening friction. The time evolution of temperature is numerically simulated when the viscosity parameter is present. Analytic results (when the viscosity is constant) with numerical calculations show that the thermal energy is controlled by the viscosity parameter. Numerical simulations (when the viscosity varies according to temperature) conduct to the time evolution of the thermal energy and temperature in the presence of linear slip-weakening friction law, thermal-pressurization (TP) friction law, softening-hardening (SH) friction law and a simple slip-weakening (SW) friction law. Results suggest that the viscosity parameter remarkably affects the temperature distribution and thermal energy due to slip-weakening friction. We have showed that the increase of temperature is due to the heat generated per unit volume and per unit time. We obtained that the temperature increase, attains certain values before decreasing and then, the system migrates toward a stationary state. In the fault, temperature distribution decreases when going far from the slip zone. The maximum temperature is obtained in the surface of slipping zone. The temperature distribution reaches the maximum when the heat generated per unit volume and unit time is maximum. This work establishes that the thermal energy is maximum at a moment when the amplitude of the seismic wave is large and that we describe as an instant of critical rupture of the seismic fault.

Acknowledgements. This paper benefits from the fruitful criticism and suggestions by the A.E. And anonymous reviewers, leading to a significant improvement of the work. They are gratefully acknowledged. We finally thank the Sector Editor Gaetano Festa.

\section{References}

Ampuero J.P. and A. M. Rubin (2008). Earthquake nucleation on rate and state faults-Agind and slip laws, J. Geophys. Res. 113, B01302; doi:10.1029/2007JB005082.

Andrews D. J. (2002). A fault constitutive relation accounting for thermal pressurization of pore fluid, J. Geophys. Res. 107, No. B12, 2363; doi:10.1029/2002JB001942.

Beeler N.M., T. E. Tullis, A. K. Kronenberg and L. A. Reinen (2007). The instantaneous rate dependence in low temperature laboratory rock friction and rock deformation experiments, J. Geophys. Res. 112, B07310; doi:10.1029/2005JB003772.

Beeler N.M., T.E. Tullis and D.L. Goldsby (2008). Constitutive relationships and physical basis of fault strength due to flash heating, J. Geophys. Res. 113, B01401; doi:10.1029/2007JB004988.

Bhattacharya A. and A. M. Rubin (2014). Frictional response to velocity steps and 1-D fault nucleation under a state evolution law with stressing-rate dependence, J. Geophys.Res. Solid Earth 119, 22722304; doi:10.1002/2013JB010671.

Blanpied M.L., T.E. Tullis and J.D. Weeks (1998). Effects of slip, slip rate, and shear heating on the friction of granite, J. Geophys. Res. 103, 489-511.

Bizzarri A. and M. Cocco (2006). A thermal pressurization model for the spontaneous dynamic rupture propagation on a three-dimensional fault, J. Geophys. Res. 111, 1-22.

Bizzarri A. (2009). Can flash heating of asperity contacts prevent melting? Geophys. Res. Lett. 36, L11304; doi:10.1029/2009GL037335.

Bizzarri A. (2010a). Toward the formulation of a realistic fault governing law in dynamic models of earthquake ruptures, in Dynamic Modelling, Edited by Alisson V. Brito, INTECH, Vienna, ISBN 978-953-7619-68-8, 167-188, http://www.sciyo.com/articles/show/title/toward-the-formulation-of-arealistic-fault-governing-law-in-dynamicmodels-of-earthquake-ruptures.

Bizzarri A. (2010b). An efficient mechanism to avert frictional melts during seismic ruptures, Earth Plan. Sci. Lett. 296, Nos. 1-2, 144 - 152; doi:10.1016/j.epsl.2010.05.012.

Bizzarri A. (2010c). Determination of the temperature field due to frictional heating on a sliding interface, rapporti Tecnici, INGV (Istituto Nazionale di Geofisica e Vulcanologia, Sezione di Bologna), 5-12. 
Bizzarri A. (2011a). Dynamic seismic ruptures on melting fault zones, J. Geophys. Res., 116, B02310; doi:10.1029/2010JB007724.

Bizzarri A. (2011b). Temperature variations of constitutive parameters can significantly affect the fault dynamics, Earth Planet. Sci. Lett. 306, 72-278; doi:10.1016/j.epsl.2011.04.009.

Bizzarri A. (2011c). On the deterministic description of earthquakes, Rev. Geophys. 49, RG3002; doi:10.1029/2011RG000356.

Bizzarri A. (2014). The Destiny of a Clast within a Molten Pseudotachylyte Vein, B. Seismol. Soc. Am. 104, No. 5, pp. 2399-2411; doi:10.1785/0120140084.

Bizzarri A. and P. Crupi (2013). Is the initial thermal state of a fault relevant to its dynamic behavior, B. Seismol. Soc. Am. 103 (3), 2062-2069; doi:10.1785/ 0120120279.

Burridge R. and L. Knopoff (1967) Model and theoretical seismicity, B. Seismol. Soc. Am. 57, 341-371.

Byerlee J.D. (1968). Brittle-ductile transition in rocks, J. Geophys. Res. 73, 4711-4750.

Byerlee J. (1978). Friction of rocks Pure Appl. Geophys. 116, 615-26.

Carlson J. M. and J. S. Langer (1989). Properties of earthquakes generated by fault dynamic Phys. Rev. Lett. 62, 22-6.

Carlson J. M. (1991). Time intervals between characteristic earthquakes and correlation with smaller events: An analysis based on a mechanical model of a fault, J. Geophys. Res. 96, 4255-4267.

Carlson J. M., J. S. Langer, B. E. Shaw and C. Tang (1991). Intrinsic properties of a Burridge-Knopoff model of an earthquake fault, Phys. Rev. A 44, 884-897.

Cao T. and K. Aki (1984). Seismicity simulation with a mass-spring model and a displacement hardening-softening friction law, Pure. Appl. Geophys. 122, 10-24.

Cao T. and K. Aki K. (1986). Seismicity simulation with a rate and state dependent friction law, Pure Appl. Geophys. $124,487-513$.

Chester F.M. and N.G. Higgs (1992). Multimechanism friction constitutive model for ultrafine quartz gouge at hydrothermal conditions, J. Geophys. Res. 97, 1859-1870.

Cochard, A. and R. Madariaga (1994). Dynamic faulting under rate-dependent friction, Pure Appl. Geophisys. 142,419-445; doi:10.1007/BF00876049.

De Lorenzo S. and M. Loddo (2009). Effect of frictional heating and thermal advection on preseismic sliding: A numerical simulation using a rate-, state- and temperature-dependent friction law. Journal of Geodynamics, Elsevier, 49 (1), pp.1.<10.1016/j.jog.2009.07.001>.<hal-00599487>.

Dieterich J.H. (1979). Modeling of rock friction 1. Experimental results and constitutive equations, J. Geophys. Res. 84, 2161-2168.

Diniega S., S. E. Smrekar, S. Anderson and E. R. Stofan (2013). The influence of temperature-dependent viscosity on lava flow dynamics, J. Geophys. Res. 118, 1516-1532; https://doi.org/10.1002/jgrf.20111.

Di Toro G., T. Hirose, S. Nielsen, G. Pennacchioni and T. Shimamoto (2006). Natural and experimental evidence of melt lubrication of faults during earthquakes, Science 311, 647-649.

Dragoni M. and S. Santini (2015). A two-asperity fault model with wave radiation, Phys. Earth Planet. Int. 248, 83-93.

Fialko Y. (2004). Temperature fields generated by the elastodynamic propagation of shear cracks in the Earth, J. Geophys. Res. 109, 1-14.

Hirose T. and T. Shimamoto (2005). Growth of molten zone as a mechanism of slip weakening of simulated faults in gabbro during frictional melting, J. Geophys. Res. 110, B05202; doi:10.1029/2004JB003207.

Jeffreys H. (1942). On the mechanics of faulting, Geol. Mag. 79, 291.

Kato N. (2001). Effect of frictional heating on pre-seismic sliding: a numerical simulation using a rate-, state- and temperature- dependent friction law, Geophys. J. Int. 147, 183-188.

Knopoff L., J.Q. Mouton and R. Burridge (1973). The dynamics of a one-dimensional fault in the presence of friction, Geophys. J. R. Astro. Soc. 35, 169-184.

Knopoff L., J.A. Landoni and M.S. Abinante (1992). Dynamical model of an earthquake fault with localization, Phys. Rev. A, 46, 7445-7449

Knopoff L. and X.X. Ni (2001). Numerical instability at the edge of a dynamic fracture, Geophys. J. Int. 147, F1-F6.

Konga G.P., F. Koumetio, D. Yemele and F. O. Djiogang (2017). One-dimensional modelling of thermal energy produced in seismic fault, J. Geophys. Eng. 14, 1639-1643.

Lachenbruch A.H., (1980). Frictional heating, fluid pressure, and the resistance to fault motion, J. Geophys. Res. 85, 6097-6122. 


\section{Guy Pascal Konga et al.}

Lee T-C and P.T. Delaney, (1987). Frictional heating and pore pressure residue to a fault slip, Geophys. J. International 88, 569-591.

Li C., S. Ouyang and M. Tang (1998). Panderivetive blown-up of ground temperature and predicting earthquake, Appl. Math. Mech. 19, 3-13.

Mase C. W. and L. Smith, (1985). Pore-fluid pressures and frictional heating on a fault surface, Pure Appl. Geophys. 122, 583-607.

Mase C. W. and L. Smith, (1987). Effects of frictional heating on the thermal, hydrologic, and mechanical response of a fault, J. Geophys. Res. 92, 6249-6272.

Nielsen S., Di Toro, G., Hirose, T., Shimamoto, T. (2008). Frictional melt and seismic slip, J. Geophys. Res. 113, B01308; doi:10.1029/2007JB005122.

Noda H., E. M. Dunham and J. R. Rice (2009). Earthquake ruptures with thermal weakening and the operation of major faults at low overall stress levels, J. Geophys. Res. 114, B07302; doi:10.1029/2008JB006143.

Nur A. (1978). Non uniform friction as a physical basis for earthquake mechanics, Pure Appl. Geophys. 116, $964-989$.

Pelap F. B., A. Fomethe, M. W. Dongmo, L. Y. Kagho, G. B. Tanekou and Y. L. Makenne (2014). Direction effects of the pulling force on the first order phase transition in a one block model for earthquakes, J. Geophys. Eng. 11, 7-13.

Qin K., Wu L.X., A. De Santis and G. Cianchini (2012). Preliminary analysis of surface temperature anomalies preceding the two major 2012 Emilia (Italy) earthquakes Ann. Geophys. 55, 823-8.

Raleigh C.B., and J. Everden, (1981). Case for low deviatoric stress in the lithosphere, in The Mechanical Behavior of Crustal Rocks, edited by N. L. Carter et al.. Geophys. Monogr. Ser., AGU, Washington, D. C vol. 24, 173 - 186.

Rezapour N., A. Bidokhti and M. Fattahi (2008). Thermal properties of the ground as an earthquake precursor, 2nd IASME/WSEAS Int. Conf. on Geology and Seismology, 63-5.

Rice J.R. (1993). Spatio-temporal complexity of slip on a fault, J. Geophys. Res. 98 (B6), 9885-9907.

Rice J.R. (2006). Heating and weakening of faults during earthquake slip, J. Geophys. Res. 111, B07302; doi:10.1029/2005JB004006.

Rice J.R., N. Lapusta and K. Ranjith (2001). Rate and state dependent friction and the stability of sliding between elastically deformable solids, J. Mech. Phys. Solids 49, 1865-1898.

Rice J. R. and M. Cocco (2006). Seismic fault rheology and earthquake dynamics, in The Dynamics of Fault Zones, 95th Dahlem Workshop (Berlin, January 2005), edited by M. R. Handy, MIT Press, Cambridge, Mass., in press.

Rubin A.M. and J. P. Ampuero (2005). Earthquake nucleation on (ageing) rate and state faults, J. Geophys. Res. 110, B11312; doi:10.1029/2005JB003686.

Ruina A.L. (1983). Slip instability and state variable friction laws, J. Geophys. Res. 88, 10, 359-10, 370.

Saraf A.K. and S. Choudhury (2004). Satellite detects surface thermal anomalies associated with the Algerian earthquakes of May 2003 Int. J. Remote Sens. 26, 2705-13.

Sleep N.H., (1995). Frictional heating and the stability of rate and state dependent frictional sliding, Geophys. Res. Lett. 22, 2785-2788.

Sibson R.H., (1973). Interaction between temperature and pore-fluid pressure during earthquake faulting: A mechanism for partial or total stress relief, Nature 243, 66-68.

Tanekou G.B., C.F. Fogang, R. Kengne and F.B. Pelap (2018). Lubrication pressure and fractional viscous damping effects on the spring-block model of earthquakes, European Physical Journal Plus 133, 150; doi:10.1140/epjp/i2018-11978-y.

Tramutoli V., Cuomo V. and Filizzola B. (2005). Assessing the potential of thermal infrared satellite surveys for monitoring seismically active areas: the case of Kocaeli (Izmit) earthquake, 17 August 1999 Remote Sens. Environ. 96, 409-26.

Turcotte D.L. and G. Schubert (1982). GEODYNAMICS6 Applications of Continuum Physics to Geological Problems, Wiley, $450 \mathrm{p}$.

Wang J.H. (1995). Effect of seismic coupling on the scaling of seismicity, Geophys. J. Int. 121, 475-488.

Wang J.H. (1996). Velocity-weakening friction law as a factor on controlling the frequency-magnitude relation of earthquake B. Seismol. Soc. Am. 86, 701-713.

Wang J.H. (1997). Effect of frictional healing on the scaling of seismicity, Geophys. Res. Lett. 24, 2527-2530.

Wang J.H. (2006). Energy release and heat generation during the 1999 Chi-Chi, Taiwan, earthquake, J. Geophys.Res. 111, B11312; doi:10.1029/2005JB004018.

Wang J.H. (2007). A dynamic study of the frictional and viscous effects on earthquake rupture: a case study 1999 Chi- 
Chi earthquake Taiwan, B. Seismol. Soc. Am. 97 (4), 1233-1244.

Wang J.H. (2011). Thermal and pore fluid pressure history on the Chelungpu fault at a depth of 1111 meters during the 1999 Chi-Chi, Taiwan, earthquake, J. Geophys. Res. 116, B03302; doi:10.1029/2010JB00 7765.

Wang J.H. (2012). Some intrinsic properties of the two-dimensional dynamical spring-model of earthquake fault, $B$. Seismol. Soc. Am. 102 (2), 822-835.

Wang J.H. (2016). Slip of one-body dynamical spring-slider model in the presence of slip-weakening friction and viscosity. Ann. Geophys. 59, (5); doi:10.4401/ag-70 63.

Wang J.H. (2017a). Slip of two-degree-of-freedom Spring-slider model in the presence of slip-dependent friction and viscosity. Ann. Geophys. 60, 6, S0659; doi:10.4401/ag-7295.

Wang J.H. (2017b). Multistable slip of a one-degree-of-freedom spring-slider model in the presence of thermalpressurized slip-weakening friction and viscosity, Nonlin. Processes Geophys. 24, 467-480, 2017; https://doi.org/10.5194/npg-24-467-2017.

Wang J.H. (2018). A study of earthquake recurrence based on a one-body spring-slider model in the presence of thermal-pressurized slip-weakening friction and viscosity, Nat. Hazards Earth Syst. Sci. 18, 1969-1983; https://doi.org/10.5194/nhess-18-1969-2018.

"CORRESPONDING AUTHOR: Fidèle Koumetio.

Unity of Research of Mechanics and Modelling of Physical Systems, Department of physics, Faculty of Science, University of Dschang, PO Box 67Dschang, Cameroon e-mail: koumetiof@yahoo.fr fidele.koumetio@univ-dschang.org (c) 2020 the Istituto Nazionale di Geofisica e Vulcanologia. All rights reserved 